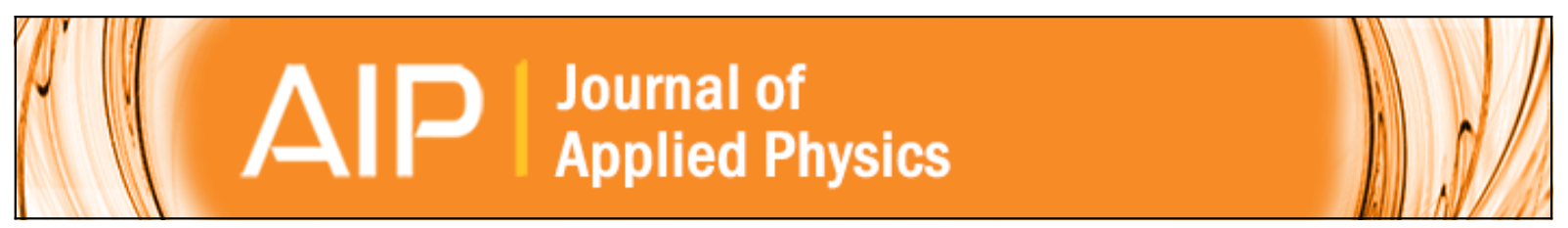

\title{
Visibility of two-dimensional layered materials on various substrates
}

M. R. Müller, A. Gumprich, E. Ecik, K. T. Kallis, F. Winkler, B. Kardynal, I. Petrov, U. Kunze, and J. Knoch

Citation: Journal of Applied Physics 118, 145305 (2015); doi: 10.1063/1.4930574

View online: http://dx.doi.org/10.1063/1.4930574

View Table of Contents: http://scitation.aip.org/content/aip/journal/jap/118/14?ver=pdfcov

Published by the AIP Publishing

\section{Articles you may be interested in}

Substrate-free layer-number identification of two-dimensional materials: A case of Mo0.5W0.5S2 alloy

Appl. Phys. Lett. 106, 223102 (2015); 10.1063/1.4921911

Enhanced absorption in two-dimensional materials via Fano-resonant photonic crystals

Appl. Phys. Lett. 106, 181104 (2015); 10.1063/1.4919760

Enhancing the visibility of graphene on GaAs

Appl. Phys. Lett. 99, 191912 (2011); 10.1063/1.3660584

Enhancing visibility of graphene on arbitrary substrates by microdroplet condensation

Appl. Phys. Lett. 97, 231905 (2010); 10.1063/1.3527081

Visibility and Raman spectroscopy of mono and bilayer graphene on crystalline silicon

Appl. Phys. Lett. 96, 081911 (2010); 10.1063/1.3323105

\section{AIP $\mid$ APL Photonics}

APL Photonics is pleased to announce Benjamin Eggleton as its Editor-in-Chief

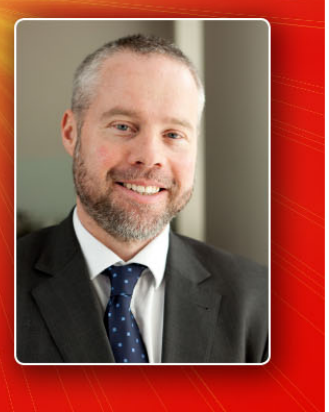




\title{
Visibility of two-dimensional layered materials on various substrates
}

\author{
M. R. Müller, ${ }^{1,2, a)}$ A. Gumprich, ${ }^{1}$ E. Ecik, ${ }^{1}$ K. T. Kallis, ${ }^{1}$ F. Winkler, ${ }^{3}$ B. Kardynal, ${ }^{3}$ I. Petrov, ${ }^{4}$ \\ U. Kunze, ${ }^{4}$ and J. Knoch ${ }^{2, a)}$ \\ ${ }^{1}$ Intelligent Microsystems Chair, TU Dortmund, Emil-Figge-Str. 68, 44227 Dortmund, Germany \\ ${ }^{2}$ Institute of Semiconductor Electronics, RWTH Aachen University, Sommerfeldstr. 24, 52074 Aachen, \\ Germany \\ ${ }^{3}$ Peter Grünberg Institute, Forschungszentrum Jülich, JARA, 52428 Jülich, Germany \\ ${ }^{4}$ Werkstoffe und Nanoelektronik, Ruhr-Universität Bochum, Universitätsstraße 150, 44801 Bochum, Germany
}

(Received 19 May 2015; accepted 27 August 2015; published online 13 October 2015)

\begin{abstract}
For the investigation of 2D layered materials such as graphene, transition-metal dichalcogenides, boron nitride, and their heterostructures, dedicated substrates are required to enable unambiguous identification through optical microscopy. A systematic study is conducted, focusing on various $2 \mathrm{D}$ layered materials and substrates. The simulated colors are displayed and compared with microscopy images. Additionally, the issue of defining an appropriate index for measuring the degree of visibility is discussed. For a wide range of substrate stacks, layer thicknesses for optimum visibility are given along with the resulting sRGB colors. Further simulations of customized stacks can be conducted using our simulation tool, which is available for download and contains a database featuring a wide range of materials. (C) 2015 AIP Publishing LLC.
\end{abstract}

[http://dx.doi.org/10.1063/1.4930574]

\section{INTRODUCTION}

Since the first investigation of the field-effect in exfoliated graphene in 2004, ${ }^{1}$ 2D layered materials have attracted a great deal of interest for the use in next-generation MOSFETs because they enable the realization of ultimately scaled transistors in a planar device architecture. ${ }^{2-19}$ In addition to graphene, several other 2D layered materials, such as transition-metal dichalcogenides (TMDCs, also for use as channel materials) and hexagonal boron nitride (h-BN, as gate insulator), have been studied in detail. Due to the fact that 2D materials offer a large variety of different band gaps, heterostructures are particularly appealing since band-gap engineering becomes feasible while maintaining the ultrathin channel layer thickness. For instance, all-2D-FETs as well as 2D heterojunction interlayer tunneling FETs have recently been investigated. ${ }^{20,21}$ However, due to a lack of appropriate epitaxial realizations, 2D heterojunctions have to be fabricated by stacking different 2D materials on top of each other using, e.g., the transfer method proposed by Dean and coworkers. ${ }^{22}$ In turn, this makes the use of intermediary substrates necessary that enable an unambiguous identification of the respective 2D materials with optical microscopy. The identification of monolayer graphene on $\mathrm{SiO}_{2} / \mathrm{Si}$ and $\mathrm{Al}_{2} \mathrm{O}_{3} /$ $\mathrm{Si}$ substrates has been subject to a number of investigations based on the mere contrast ${ }^{23}$ or on the CIE (Commission Internationale de l'Éclairage) color spaces ${ }^{24,25}$ to account for the color perception of the human eye. In contrast, the visibility of TMDCs, h-BN, and in particular, heterostructures as well as further promising substrates has so far not been studied comprehensively. ${ }^{26-28}$

\footnotetext{
a) Authors to whom correspondence should be addressed. Electronic addresses: marcelrene.mueller@tu-dortmund.de, Tel.: +49 231 755-4301, Fax: -4450 and knoch@iht.rwth-aachen.de, Tel.: +49 241 802-7890, Fax: -2246
}

Here, we present results on the optimum visibility of various 2D layered materials and heterostructures thereof on substrates typically used in experiments. Our results show that the standard practice of employing a substrate with, e.g., $280 \mathrm{~nm} \mathrm{SiO}_{2}$ on $\mathrm{Si}$ as appropriate for monolayer graphene $^{29,30}$ is not optimal for the visibility of TMDCs or h$\mathrm{BN}$. Therefore, our study provides guidelines how to prepare optimum (intermediary) substrates for the investigation of devices based on 2D layered materials.

\section{CALCULATION MODEL}

Figure 1 provides an overview on the calculation model, which can be divided into three parts. (1) First, the refraction spectra $S(\lambda)$ are calculated separately for each stack to be investigated, e.g., $\mathrm{SiO}_{2} / \mathrm{Si}$ and $\mathrm{WSe}_{2}$ on $\mathrm{SiO}_{2} / \mathrm{Si}$, respectively, called the "background" (BG) and the "foreground" (FG) in the following. The calculation is based on the Fresnel formalism and includes angular dependency of the incident light defined by the numeric aperture (NA) of the object lens in use. This approach has already been employed several times ${ }^{23-25}$ and is presented in detail in Appendix A. Additionally, we gathered, employed, and compared various literature sources for the optical properties $(n, k)$ characterizing the materials under investigation; the data are mostly wavelength-dependent and its sources are given in Appendix B. In case of the 2D layered materials, the literature sources of the interlayer distances used are listed additionally. Note that the Fresnel formalism assumes homogeneous layers, ideal interfaces, and is only valid for wavelengths much larger than atomic distances. ${ }^{31}$

(2) After determination of the refraction spectra, they are used to calculate the CIEXYZ colors, which represent the colors perceived by the human eye. The transformation consists of an integration over wavelengths including the light source spectrum $L(\lambda)$ and the so-called color matching 


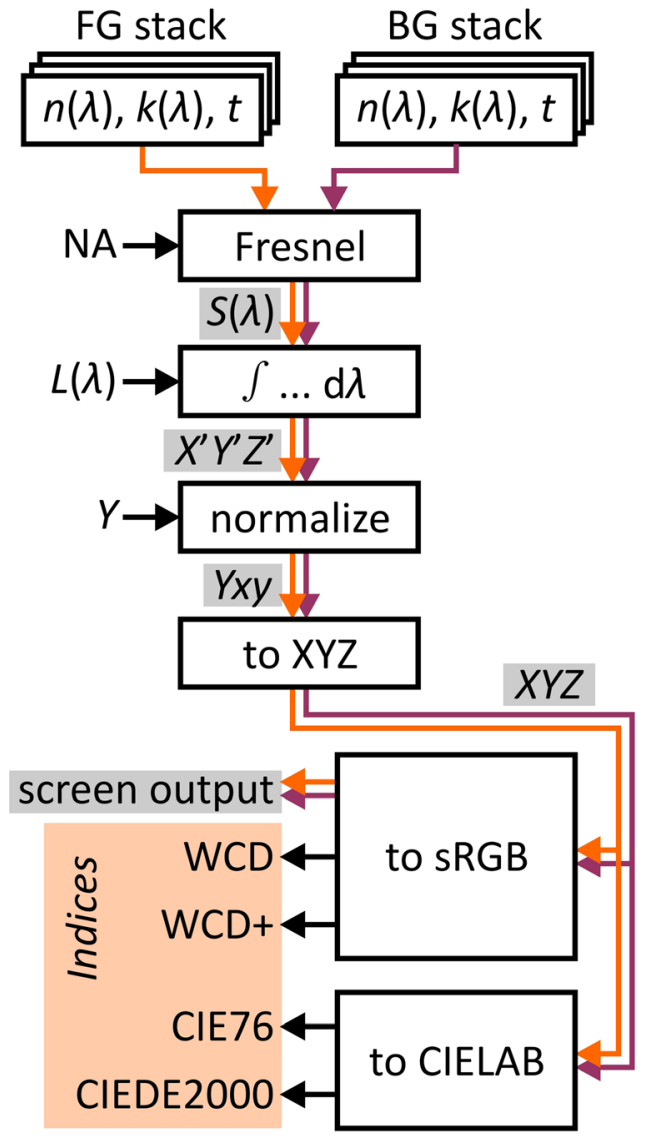

FIG. 1. Flow diagram of the entire calculation model.

functions. The resulting intermediary $X^{\prime} Y^{\prime} Z^{\prime}$ values are transformed to the $Y x y$ color space in order to introduce a global luminance $Y$, before being translated into the final $X Y Z$ values. This is described in detail in Appendix $\mathrm{C}$.

(3) In the final step, the $X Y Z$ colors are converted into the sRGB and CIELAB color spaces using standard transformations. The sRGB values are used to visualize the stacks' colors on the screen, and the SRGB and CIELAB values are employed to calculate visibility indices which serve to assess and compare the degree of visibility. These indices are discussed in detail in Section IV.

\section{EVALUATION OF THE MODEL WITH $\mathrm{SiO}_{2}$ ON Si AND WSe $\mathrm{ON} \mathrm{SiO}_{2} / \mathrm{Si}$}

In order to evaluate the described model, $\mathrm{SiO}_{2}$ on $\mathrm{Si}$ stacks with $\mathrm{SiO}_{2}$ thicknesses of 0 (bare $\mathrm{Si}$ ), 86, 175, and $272 \mathrm{~nm}$, verified with ellipsometry, are fabricated by wet thermal oxidation of (100) Si wafers. Optical microscopy images are taken on a digital Keyence VHX-600 microscope with a VH-Z500 object lens. For all images taken, the same microscopy hardware and software settings are used, and all image enhancement features are switched off. A gamma correction factor of 2.3 is set to match the standard gamma correction of the sRGB color space, and a standardized grey card is used to perform a white balance. The remaining parameters to be chosen are the magnification and the exposure time. The extracted RGB (red-green-blue) colors from the microscopy images taken are presented in Fig. 2 along with



FIG. 2. Colors extracted from microscopy images vs. simulations for x nm $\mathrm{SiO}_{2}$ on $\mathrm{Si}$.

the simulated colors, where the $\mathrm{x}$-axis represents the thickness of the $\mathrm{SiO}_{2}$ layer.

The simulations are performed with an NA $=0.82$ taken from the data sheet of the object lens. ${ }^{32} Y=0.37$ is set to match the luminosity of the background. Due to the described white balancing of the microscope, simulations employing the standard type D65 daylight as light source are found to match the colors of the images best. This approach fits the microscopy images better than disabling the white balance and simulating with the source spectrum of the microscope light source in use, a halogen lamp with $T=3100 \mathrm{~K}$. Fig. 2 indicates that the reproduction of the colors is excellent. Imperfections are attributed to

- the modeling of the light source intensity angular distribution, which might not follow the assumed Gaussian distribution,

- the calibration of light source and white balancing might be non-ideal,

- the complex digital microscopy hardware/software system, which cannot be fully modeled, and

- literature values of optical properties $(n, k)$ may differ from those of the material under investigation, especially for atomically thin films.

Despite these imperfections, our simulations also yield very good results for more complex stacks, exemplary for $\mathrm{WSe}_{2}$ on $287 \mathrm{~nm} \mathrm{SiO} / \mathrm{Si}$ : For comparison, we exfoliated $\mathrm{WSe}_{2}$ flakes, took microscopy images, and performed atomic force microscopy (AFM) to measure the heights the flakes. Fig. 3(a) shows microscopy images of two flakes with their thicknesses identified either in $\mathrm{nm}$, or, for thin sections by the number of layers counted by using the AFM surface profile (one exemplary shown in the figure). For the interlayer distance, the literature value $6.7 \AA$ (Ref. 28) is used. Note that the distance of the first layer to the surface is larger than the interlayer distance which is attributed to the $\mathrm{SiO}_{2}-\mathrm{WSe}_{2}$ interface; both monolayers shown were confirmed by photoluminescence measurements. Fig. 3(b) shows the colors extracted from the respective sections in the images and plotted on a thickness scale. Fig. 3(c) displays the simulations performed, with $Y=0.17$ chosen to match the background's luminance. The color sequence: violet-blue-grey-yellow-red of microscopy images and simulations is in agreement. However, the blue and red colors are more pronounced, and the grey colors appear darker in the simulations. We also investigated $\mathrm{h}-\mathrm{BN}$ on $32 \mathrm{~nm}$ $\mathrm{SiO}_{2} / \mathrm{Si}$ (not shown) and found similar excellent accordance of microscopy images and simulations. 
(a) Optical microscopy images mag $=5 \mathrm{kx}, 1 / 60 \mathrm{~s}$

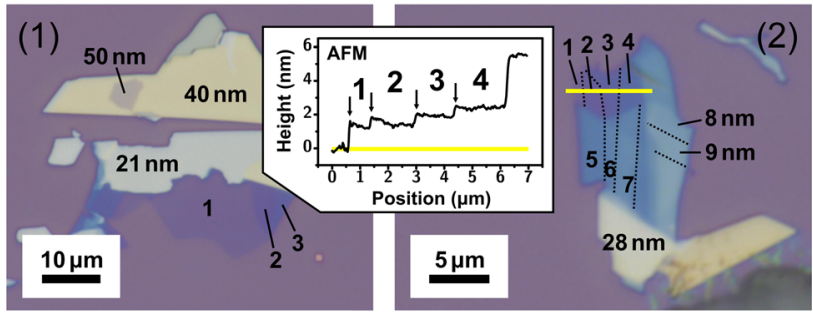

(b) Extracted from images

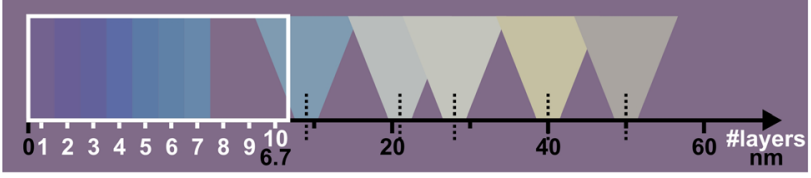

(c) Simulation

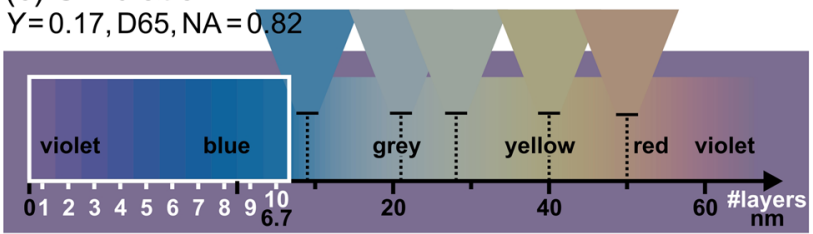

FIG. 3. Microscopy images vs. simulations for $\mathrm{n}$ layers/x nm $\mathrm{WSe}_{2}$ on $\mathrm{SiO}_{2} / \mathrm{Si}$.

For $\mathrm{WSe}_{2}$, the impact of strongly deviating optical properties $(n, k)$ for atomically thin films was also considered. In particular, we compared the color rendering of $(n, k)$ data from a $2.8 \mathrm{~nm}$ thin $\mathrm{WSe}_{2}$ flake $^{33}$ with those of bulk material $^{34}$ and found no substantial difference. However, the source of the $(n, k)$ values can generally have a strong effect for other materials, e.g., h-BN.

\section{VISIBILITY INDICES}

The goal of this study is to provide guidelines how to prepare optimum substrates for the investigation of 2D layered materials. To this end, an important issue is to define an appropriate index for measuring the degree of visibility. One index used in the past is the CIE76- $\Delta E$ index $^{24}$

$$
\Delta E_{\mathrm{CIE76}}=\sqrt{\left(\Delta L^{*}\right)^{2}+\left(\Delta a^{*}\right)^{2}+\left(\Delta b^{*}\right)^{2}}
$$

which is based on the differences $\Delta$ of the CIELAB colors between foreground and background, e.g., $\Delta L^{*}=L_{\mathrm{FG}}^{*}-L_{\mathrm{BG}}^{*}$. We find that this index and its improvement, the CIEDE2000- $\Delta E$ index, does not perfectly describe the perceived contrast on computer screens, e.g., visibility values of yellow and green colors are too high (cf. Fig. 4). Being based on CIELAB colors, these indices should be optimized to describe the perceived contrast from a direct observation in a microscope. In order to describe contrasts perceived on a computer screen, we have earlier proposed a weighted color difference (WCD) index ${ }^{25}$

$$
\mathrm{WCD}=\sqrt{1.89(\Delta R)^{2}+8.68(\Delta G)^{2}+0.11(\Delta B)^{2}}
$$

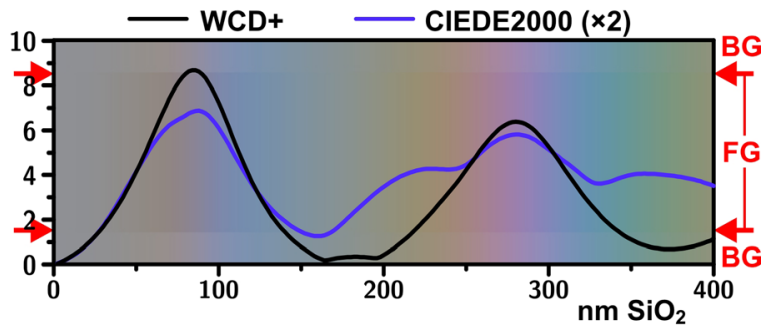

FIG. 4. wcd + and CIEDE2000 (with a factor 2) indices (both with $\mathrm{Y}_{\mathrm{BG}}=0.28$ ) for a graphene monolayer on $\mathrm{x} \mathrm{nm} \mathrm{SiO}_{2} / \mathrm{Si}$. The corresponding background and foreground colors are also shown.

which is based on the differences $\Delta$ of the R, G, and B channels between foreground and background, e.g., $\Delta R=R_{\mathrm{FG}}-R_{\mathrm{BG}}$. However, the index delivers too high values for cyan colors, e.g., for a graphene monolayer on $\sim 335 \mathrm{~nm} \mathrm{SiO}_{2} / \mathrm{Si}$. This can be compensated by weighting the $\Delta R$ term with a factor

$$
\alpha_{R}=\left(R_{\mathrm{FG}}+R_{\mathrm{BG}}\right)(2 \cdot 255)^{-1},
$$

because the perceived red contrast increases along with the absolute values $\left(R_{\mathrm{FG}}, R_{\mathrm{BG}}\right)$ of the red channel. Another important issue which has to date not been considered in discussions is the selection of an equal luminance level for each background in order to assure comparability, because the luminance level strongly influences the perceived contrast. We therefore propose the following improved WCD+ index:

$$
\begin{aligned}
\mathrm{WCD}+\left(Y_{\mathrm{BG}}=0.28\right)= & 0.21 \alpha_{R}|\Delta R|+0.72|\Delta G| \\
& +0.07|\Delta B|,
\end{aligned}
$$

where the weighting factors are based on the luminance contributions of the RGB channels $(Y=0.21 R+0.72 G+0.07 B)$. Note that the factors in the equation capture the fact that the contrast seen by humans is mainly determined by the contrast of green tones and almost not at all by blue tones. We chose the background to always possess the luminosity $Y_{\mathrm{BG}}=0.28$, which equals a CIELAB luminosity of $L=60$. This is somewhat lighter than neutral grey $(L=50)$, which appears rather dark on computer screens (in the sRGB color space). Fig. 4 exemplary presents the WCD + and the CIEDE2000 (with a factor 2) indices (both with $Y_{\mathrm{BG}}=0.28$ ) for a graphene monolayer on $x \mathrm{~nm} \mathrm{SiO} / 2 / \mathrm{Si}$ for comparison. The resulting colors of the background and the foreground (region between the red arrows) are also shown.

\section{VISIBILITY SIMULATIONS OF 2D LAYERED MATERIALS}

Table I provides an overview on the visibility simulations of 2D layered materials and heterostructures on various substrates. Each field in the table contains: (1) sRGB colors of background and foreground of two selected WCD+ optima, (2) the respective layer thicknesses and WCD+ values, and (3) two selected CIEDE2000 optima (all with $\left.Y_{\mathrm{BG}}=0.28\right)$. " $x$ " denotes the thickness varied in order to obtain the optimum visibility. Note that printed colors (CMYK color space) may be altered. We base our evaluation 
TABLE I. Optimum thicknesses of substrates for the visibility of graphene, TMDCs, h-BN, and heterostructures. Each field in the table contains: (1) sRGB colors of the WCD + optima, top: background, bottom: foreground, (2) (local) optimal thickness $\left(x_{\mathrm{opt}}\right)$ \{WCD +$\}$, and (3) (local) optimal thickness $\left(x_{\mathrm{opt}}\right)$ $\{$ CIEDE2000\}.



${ }^{1}$ Foreground $=2 \mathrm{D}$ layered material $(2 \mathrm{DLM})$ stack + background.

${ }^{2}$ Transfer stack as originally specified by Dean et al.: ${ }^{22}$ PMMA $950 \mathrm{~K}, 4 \%$ (spin-on velocity $4000 \mathrm{rpm} \rightarrow 290 \mathrm{~nm}$ ) + PVA $9 \mathrm{~K}$, $4 \%$ (spin-on velocity $4000 \mathrm{rpm}$ $\rightarrow 70 \mathrm{~nm})$.

${ }^{3}$ One layer.

${ }^{4}$ Ten layers.

${ }^{5} \mathrm{Bare} \mathrm{Si}$.

${ }^{6} \mathrm{NA}=0.44$.

${ }^{7} \mathrm{NA}=0.43$.

on the presented WCD+ index; however, the CIEDE2000 is given for comparison. As the values in the table indicate, WCD+ and CIEDE2000 optima are mostly consistent and differ only in some case. In these cases, we still find the CIEDE2000 to yield high visibility for the optima of the $\mathrm{WCD}+$ index.

We observe a common color sequence for all 2D layered material on oxides, which is grey-brown-light blue-greyorange-pink-cyan (cf. Fig. 2) for 0 to $\sim 300 \mathrm{~nm}$ oxide thickness. This explains why usually two optima are found, one at $\sim 50-100 \mathrm{~nm}$ and one at $\sim 200-300 \mathrm{~nm}$. We also find that the optima are usually in the region of a color swing within the color sequence. Note that the width of the visibility optima is always large enough (at least $\gtrsim 20 \mathrm{~nm}$ ) to allow for variations due to the manufacturing process (see the visibility curve in Fig. 4).

Further investigated is the optimum visibility as a function of the thickness of the 2D layered material. As a general trend, we find that an increase of the thickness of the 2D layered material reduces the optimum thicknesses of the oxide. For example, one layer $(1 \mathrm{~L})$ of $\mathrm{WSe}_{2}$ is best visible on $\mathrm{SiO}_{2} /$ $\mathrm{Si}$ with an oxide thicknesses of $73 \mathrm{~nm}$ and $268 \mathrm{~nm}$, whereas in the case of ten layers $(10 \mathrm{~L})$ the optima shift to $44 \mathrm{~nm}$ and $243 \mathrm{~nm}$, respectively (cf. Table I).
Table I also contains visibility optima for a transfer stack (polymethyl methacrylate (PMMA)/polyvinyl alcohol (PVA)/ $\mathrm{SiO}_{2} / \mathrm{Si}$ ) as proposed by Dean and co-workers. ${ }^{22}$ In contrast to their recommendation of using bare $\mathrm{Si}\left(\mathrm{no} \mathrm{SiO}_{2}\right)$, we find optimum $\mathrm{SiO}_{2}$ thicknesses of $106 \mathrm{~nm}$ and $300 \mathrm{~nm}$. Furthermore, Table I displays simulations of heterostructures. As an example, a graphene $(1 \mathrm{~L}) / \mathrm{h}-\mathrm{BN}(8 \mathrm{~L}) / \mathrm{MoS} 2(1 \mathrm{~L})$ structure is included, which can be used as an all-2D-FET setup. ${ }^{20}$ Additionally, an h-BN (30 L)/graphene $(1 \mathrm{~L}) / \mathrm{h}-\mathrm{BN}(30 \mathrm{~L})$ is shown, where the boron nitride is used to improve the electrical properties of the graphene. ${ }^{35}$

Table I presents a number of typical TMDCs, substrates, and combinations in order to show that dedicated (intermediary) substrates yield strongly improved visibilities. However, our simulation tool is available for download to simulate a substantially larger variety of other TMDCs and substrates. ${ }^{36}$

\section{SUMMARY AND CONCLUSION}

A simulation study of the visibility of 2D layered materials, such as graphene, transition-metal dichalcogenides, and boron nitride - and their heterostructures-on various substrates is presented. For the realization of the simulations, a software is created, which contains a database with optical 
properties of a wide range of materials. For a stack of choice, the software calculates and displays the sRGB colors along with the newly designed WCD + visibility index allowing the choice of layer thicknesses for optimum visibility. The simulation results are compared with experimentally prepared stacks, and excellent reproduction of the colors is found. For typical 2D layered materials and substrates, optimum layer thicknesses and the respective colors are presented. This data, along with the software which is available for download, can be used to compile stacks with optimum visibility for each material to be investigated.

\section{ACKNOWLEDGMENTS}

The authors would like to thank Dr. D. Truhn for his help with the modeling. The authors particularly thank the German Research Foundation (DFG) for their funding (Grant No. KN545/3-2).

\section{APPENDIX A: CALCULATION OF THE REFRACTION SPECTRA}

The refraction spectra are calculated by employing the Fresnel formalism in matrix notation. Figure 5 illustrates that a stack with layers $0 \ldots N$ is assumed, where layer 0 is air and layer $N$ is the substrate.

Electromagnetic waves travelling forward $\left(E_{F}\right)$ and backward $\left(E_{B}\right)$ can be described as follows: ${ }^{37}$

$$
\begin{aligned}
\left(\begin{array}{l}
E_{F}\left(x_{0}^{-}\right) \\
E_{B}\left(x_{0}^{-}\right)
\end{array}\right) & =\mathcal{M}\left(\begin{array}{l}
E_{F}\left(x_{N-1}^{+}\right) \\
E_{B}\left(x_{N-1}^{+}\right)
\end{array}\right), \\
\mathcal{M} & =T_{01} P_{1} T_{12} P_{2} \ldots T_{(N-1) N} .
\end{aligned}
$$

The notation includes a layer-to-layer ( $l$ to $m$ ) transfer matrix

$$
T_{l m}=\frac{1}{t_{l m}}\left(\begin{array}{cc}
1 & r_{l m} \\
r_{l m} & 1
\end{array}\right)
$$

and a within-layer propagation matrix

$$
P_{l}=\left(\begin{array}{cc}
\mathrm{e}^{+i 2 \pi \lambda^{-1} \tilde{n}_{l} \tau_{l} \cos \phi_{l}} & 0 \\
0 & \mathrm{e}^{-i 2 \pi \lambda^{-1} \tilde{n}_{l} \tau_{l} \cos \phi_{l}}
\end{array}\right),
$$

where $\lambda$ is the wavelength, $\tilde{n}_{l}=n_{l}-i k_{l}$ is the complex refraction index, and $\tau_{l}$ is the thickness of layer $l$. The angle of incidence $\phi_{l}$ is calculated recursively, from $\phi_{0}$ (known) to $\phi_{N}$, by Snell's law

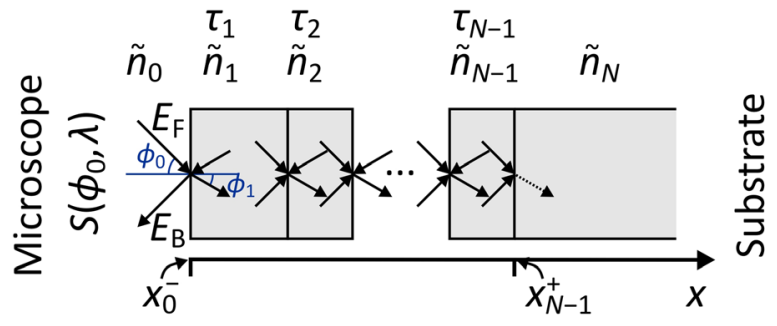

FIG. 5. Complex refraction indices $\tilde{n}_{l}$ and layer thicknesses $\tau_{l}$ of a multilayer stack are used to calculate its refraction spectrum $S\left(\phi_{0}, \lambda\right)$ depending on the angle of incidence $\phi_{0}$.

$$
\sin \phi_{l} \Re\left(\tilde{n}_{l}\right)=\sin \phi_{m} \Re\left(\tilde{n}_{m}\right) .
$$

The transmission and reflection coefficients depend on the polarization of the incident wave and are given by:

- TE polarization

$$
\begin{aligned}
r_{l m} & =\frac{\tilde{n}_{l} \cos \phi_{l}-\tilde{n}_{m} \cos \phi_{m}}{\tilde{n}_{l} \cos \phi_{l}+\tilde{n}_{m} \cos \phi_{m}}, \\
t_{l m} & =\frac{2 \tilde{n}_{l} \cos \phi_{l}}{\tilde{n}_{l} \cos \phi_{l}+\tilde{n}_{m} \cos \phi_{m}} .
\end{aligned}
$$

- TM polarization

$$
\begin{aligned}
r_{l m} & =\frac{\tilde{n}_{m} \cos \phi_{l}-\tilde{n}_{l} \cos \phi_{m}}{\tilde{n}_{m} \cos \phi_{l}+\tilde{n}_{l} \cos \phi_{m}}, \\
t_{l m} & =\frac{2 \tilde{n}_{l} \cos \phi_{l}}{\tilde{n}_{m} \cos \phi_{l}+\tilde{n}_{l} \cos \phi_{m}} .
\end{aligned}
$$

From Eq. (A1), the refraction (power) spectrum can be calculated using $E_{B}\left(x_{N-1}^{+}\right)=0$ (no backward-travelling wave from the substrate) and elimination of $E_{F}\left(x_{N-1}^{+}\right)$

$$
S\left(\phi_{0}, \lambda\right)=\left(\frac{E_{B}\left(x_{0}^{-}\right)}{E_{F}\left(x_{0}^{-}\right)}\right)^{2}=\left(\frac{\mathcal{M}_{12}}{\mathcal{M}_{11}}\right)^{2} .
$$

Afterwards, the input angles are integrated according to

$$
S(\lambda)=\int_{0}^{\phi_{0}^{\max }} \mathrm{d} \phi_{0} S\left(\phi_{0}, \lambda\right) N\left(0,\left(\phi_{0}^{\max }\right)^{2}\right),
$$

with $\phi_{0}^{\max }=\sin ^{-1} \mathrm{NA}$, and a weighting normal distribution $N\left(0,\left(\phi_{0}^{\max }\right)^{2}\right)$ with mean 0 and standard deviation $\phi_{0}^{\max }$ used to model the angular light power distribution of the object lens. ${ }^{38}$ The spectra are calculated separately for both polarizations, TE and TM. Under the simplifying assumption of unpolarized incident light, the total spectrum is calculated by adding the two spectra

$$
S^{\mathrm{unpol}}(\lambda)=S^{\mathrm{TE}}(\lambda)+S^{\mathrm{TM}}(\lambda)
$$

Note that in the simulations

- step sizes of $\Delta \phi=1^{\circ}, \Delta \lambda=2 \mathrm{~nm}$ are used, and

- total reflection is avoided by ending the simulations at the maximum angle without total reflection. In such a case, the maximum $\mathrm{NA}^{\max }$ is denoted.

\section{APPENDIX B: LIST OF SOURCES OF OPTICAL PROPERTIES AND INTERLAYER DISTANCES OF THE MATERIALS IN USE}

\begin{tabular}{lcc}
\hline \hline Material & Source for $(n, k)$ & Interlayer distance $(\AA)$ \\
\hline Graphene & 39 & 3.35 (Ref. 45) \\
$\mathrm{WSe}_{2}$ & 33 and 34 & 6.7 (Ref. 28) \\
$\mathrm{MoS}_{2}$ & 40 & 6.15 (Ref. 46) \\
b-BN & 41 & 3.33 (Ref. 47) \\
$(100) \mathrm{Si}$ & 42 & \\
\hline
\end{tabular}


(Continued.)

\begin{tabular}{lcc}
\hline \hline Material & Source for $(n, k)$ & Interlayer distance $(\AA)$ \\
\hline${\text { Therm. } \mathrm{SiO}_{2}}_{\mathrm{Al}_{2} \mathrm{O}_{3}}$ & 42 \\
$\mathrm{HfO}_{2}$ & 43 \\
$\mathrm{Al}$ & 41 \\
PMMA 950 K 4\% & $A=1.472, B=0.00372^{\mathrm{a}}$ \\
PVA 9 K 4\% & $A=1.506, B=0.00271^{\mathrm{a}}$ \\
\hline \hline
\end{tabular}

${ }^{\text {a } O b t a i n e d ~ b y ~ a ~ C a u c h y ~ f i t ~ f r o m ~ e l l i p s o m e t r i c ~ m e a s u r e m e n t s: ~}$ $n\left(\frac{\lambda}{\mu \mathrm{m}}\right)=A+B\left(\frac{\lambda}{\mu \mathrm{m}}\right)^{-2}, k=0$.

\section{APPENDIX C: CALCULATION OF THE CIEXYZ COLORS}

Our calculation model supports the simulation of several stacks, e.g., the simulation of $1 . . . n$ layers of $\mathrm{WSe}_{2}$ on $\mathrm{SiO}_{2} /$ $\mathrm{Si}$. In this example, only the foreground is varied, and $n+1$ stacks are calculated ( $n$ foregrounds and one background). If the background is varied, $2 n$ stacks are calculated. The flow diagram in Fig. 1 illustrates the calculation approach. First, the spectrum $S(\lambda)$ is converted into intermediary CIEXYZ colors

$$
\left(\begin{array}{c}
X_{j}^{\prime} \\
Y_{j}^{\prime} \\
Z_{j}^{\prime}
\end{array}\right)=\int_{380 \mathrm{~nm}}^{780 \mathrm{~nm}} S_{j}(\lambda) L(\lambda)\left(\begin{array}{c}
\bar{x}(\lambda) \\
\bar{y}(\lambda) \\
\bar{z}(\lambda)
\end{array}\right) \mathrm{d} \lambda,
$$

where $j$ is the index of the stack, $L(\lambda)$ is the light source spectrum, and $\bar{x}(\lambda), \bar{y}(\lambda)$, and $\bar{z}(\lambda)$ are the CIE 1931 color matching functions. ${ }^{48} \mathrm{We}$ employed two different light source spectra: (1) A standard type D daylight illuminant D65 (Ref. 48) and (2) the spectrum of a black body following Planck's law:

$$
L(\lambda) \sim\left(\frac{\lambda}{\mathrm{nm}}\right)^{-5}\left[\exp \left(\frac{1.44 \times 10^{7} \mathrm{~K} \mathrm{~nm}}{T \lambda}\right)-1\right]^{-1},
$$

which enables the simulation of, e.g., a type A illuminant, i.e., tungsten-filament lighting $(\mathrm{T} \sim 3100 \mathrm{~K})$.

The calculated values are not the final XYZ colors because the luminosity is yet to be set correctly. To this end, the values are normalized according to

$$
\left(\begin{array}{c}
x_{j} \\
y_{j}
\end{array}\right)=\frac{1}{X_{j}^{\prime}+Y_{j}^{\prime}+Z_{j}^{\prime}}\left(\begin{array}{c}
X_{j}^{\prime} \\
Y_{j}^{\prime}
\end{array}\right)=: \frac{1}{k_{j}}\left(\begin{array}{c}
X_{j}^{\prime} \\
Y_{j}^{\prime}
\end{array}\right) .
$$

Note that $z$ is implicitly given due to $z=1-x-y$. These values are complemented to obtain the Yxy color by introducing the luminosity $Y$, which is set by default to $\left(\frac{60+16}{116}\right)^{3} \approx 0.28$, hence, equaling a CIELAB luminosity of $L=60 . Y=0$ represents black, while $Y=1$ yields a color on the $x y$-plane containing the reference white point (D65). Our software includes a controller, which allows tuning $Y$ to adjust the luminosity in real-time during the visualization of the colors.
Once the Yxy color is set, standard transformations can be used to convert the color to any other color space. In our case, we first convert to CIEXYZ by

$$
\left(\begin{array}{c}
X_{j} \\
Y_{j} \\
Z_{j}
\end{array}\right)=\frac{k_{j}}{k_{\mathrm{ref}}} \cdot \frac{Y}{y_{\mathrm{ref}}}\left(\begin{array}{c}
x_{j} \\
y_{j} \\
1-x_{j}-y_{j}
\end{array}\right) .
$$

Note that in order to maintain the correct relations of luminosities of all involved stacks $j$, they are multiplied with a factor $\frac{k_{j}}{k_{\text {ref }}} \cdot \frac{1}{y_{\text {ref }}}$ instead of $\frac{1}{y_{j}}$.

${ }^{1}$ K. S. Novoselov et al., Science 306(5696), 666-669 (2004).

${ }^{2}$ M. L. Tsai et al., ACS Nano 8, 8317 (2014).

${ }^{3}$ G. Prasad and O. N. Srivastava, J. Phys. D: Appl. Phys. 21, 1028 (1988).

${ }^{4}$ A. Pospischil et al., Nat. Nanotechnol. 9, 257 (2014).

${ }^{5}$ W. Zhang et al., Sci. Rep. 4, 3826 (2014).

${ }^{6}$ M. R. Esmaeili-Rad and S. Salahuddin, Sci. Rep. 3, 2345 (2013).

${ }^{7}$ O. L. Sanchez et al., Nat. Nanotechnol. 8, 497 (2013).

${ }^{8}$ B. Baugher et al., Nat. Nanotechnol. 9, 262 (2014).

${ }^{9}$ W. Choi et al., Adv. Mater. 24, 5832 (2012).

${ }^{10}$ D. J. Late et al., ACS Nano 7, 4879 (2013).

${ }^{11}$ F. K. Perkins et al., Nano Lett. 13, 668 (2013).

${ }^{12}$ D. Jena, Proc. IEEE 101, 1585 (2013).

${ }^{13}$ G. Fiori et al., Nat. Nanotechnol. 9, 768 (2014).

${ }^{14}$ W. S. Hwang et al., Appl. Phys. Lett. 101, 013107 (2012).

${ }^{15}$ B. Radisavljevic et al., Nat. Nanotechnol. 6, 147 (2011).

${ }^{16}$ S. Fathipour et al. Appl. Phys. Lett. 106, 022114 (2015).

${ }^{17}$ S. Fathipour et al., Appl. Phys. Lett. 105, 192101 (2014).

${ }^{18}$ S. Das and J. Appenzeller, Appl. Phys. Lett. 103, 103501 (2013).

${ }^{19}$ S. Fathipour et al., in 2013 Device Research Conference (DRC), pp. $115-116$.

${ }^{20}$ T. Roy et al., ACS Nano 8(6), 6259-6264 (2014).

${ }^{21}$ M. O. Li et al., IEEE J. Electron Devices Soc. 3, 200 (2015).

${ }^{22}$ C. R. Dean et al., Nat. Nanotechnol. 5, 722-726 (2010).

${ }^{23}$ P. Blake et al., Appl. Phys. Lett. 91, 063124 (2007).

${ }^{24}$ L. Gao et al., ACS Nano 2(8), 1625-1633 (2008).

${ }^{25}$ C. Kontis et al., Appl. Opt. 51(3), 385-389 (2012).

${ }^{26}$ H. Li et al., ACS Nano 7(11), 10344-10353 (2013).

${ }^{27}$ R. V. Gorbachev et al., Small 7(4), 465-468 (2011).

${ }^{28}$ M. M. Benameur et al., Nanotechnology 22(12), 125706 (2011).

${ }^{29}$ A. Splendiani et al., Nano Lett. 10(4), 1271-1275 (2010).

${ }^{30}$ N. R. Pradhan et al., Appl. Phys. Lett. 102(12), 123105 (2013).

${ }^{31}$ O. S. Heavens, Rep. Prog. Phys. 23, 1 (1960).

${ }^{32}$ See http://www.keyence.com for Modellreihe VH lens Objektive (für digitale Mikroskope) Katalog (Deutsch), VH_Lens_GC_622622 _DE_10441.pdf (accessed April 1, 2015).

${ }^{33}$ S. M. Eichfeld et al., APL Mater. 2, 092508 (2014).

${ }^{34}$ A. R. Beal et al., J. Phys. C: Solid State Phys. 9(12), 2449 (1976).

${ }^{35}$ S. Engels et al., Phys. Status Solidi B 251(12), 2545-2550 (2014).

${ }^{36} \mathrm{See}$ http://www.iht.rwth-aachen.de/research/downloads for VS.Lab software.

${ }^{37}$ See http://www.photonics.intec.ugent.be/download/ocs129.pdf for a detailed derivation of the Fresnel formalism in matrix notation (accessed April 1, 2015).

${ }^{38}$ M. Bruna and S. Borini, J. Phys. D: Appl. Phys. 42, 175307 (2009).

${ }^{39}$ J. W. Weber et al., Appl. Phys. Lett. 97, 091904 (2010).

${ }^{40}$ A. R. Beal et al., J. Phys. C: Solid State Phys. 12(5), 881 (1979).

${ }^{41}$ J. A. Woollam CompleteEASE Database.

${ }^{42}$ C. M. Herzinger et al., J. Appl. Phys. 83(6), 3323-3336 (1998).

${ }^{43}$ T. Lichtenstein, Handbook of Thin Film Materials (College of Engineering and Applied Science, University of Rochester, 1979).

${ }^{44}$ E. D. Palik, Handbook of Optical Constants of Solids, 1st ed. (Academic Press, 1997), pp. 369-406.

${ }^{45}$ P. Mukhopadhyay and R. K. Gupta, Graphite, Graphene, and Their Polymer Nanocomposites (CRC Press, 2012).

${ }^{46}$ T. Böker et al., Phys. Rev. B 64(23), 235305 (2001).

${ }^{47}$ Y. Shi et al., Nano Lett. 10(10), 4134-4139 (2010).

${ }^{48}$ See http://files.cie.co.at/204.xls for Selected Colorimetric Tables (accessed April 17, 2015). 